\title{
Electromagnetic considerations for communicating on correlated MIMO channels with covariance information
}

Michael A. Jensen

jensen@byu.edu

Jon W. Wallace

wall@ieee.org

Follow this and additional works at: https://scholarsarchive.byu.edu/facpub

Part of the Electrical and Computer Engineering Commons

\section{Original Publication Citation}

Wallace, J. W., and M. A. Jensen. "Electromagnetic Considerations for Communicating on Correlated MIMO Channels with Covariance Information." Wireless Communications, IEEE Transactions on 7.2 (28): 543-51

\section{BYU ScholarsArchive Citation}

Jensen, Michael A. and Wallace, Jon W., "Electromagnetic considerations for communicating on correlated MIMO channels with covariance information" (2008). Faculty Publications. 207.

https://scholarsarchive.byu.edu/facpub/207

This Peer-Reviewed Article is brought to you for free and open access by BYU ScholarsArchive. It has been accepted for inclusion in Faculty Publications by an authorized administrator of BYU ScholarsArchive. For more information, please contact ellen_amatangelo@byu.edu. 


\title{
Electromagnetic Considerations for Communicating on Correlated MIMO Channels with Covariance Information
}

\author{
Jon W. Wallace, Member, IEEE, and Michael A. Jensen, Fellow, IEEE
}

\begin{abstract}
Previous results for correlated block-fading MIMO channels with covariance information indicate guaranteed capacity growth with additional transmit elements and that in rapidly fading channels, vanishing element spacing maximizes capacity. However, because prior analysis neglects antenna electromagnetic coupling, the observations are not necessarily valid for small inter-element spacing. This work applies radiated power considerations to the analysis to demonstrate that additional elements do not always increase capacity and that vanishing element spacing is not optimal. An effective gain metric is introduced that quantifies the performance increase with additional transmitters in the presence of transmit correlation and mutual coupling. Performance simulations using the electromagnetic properties of uniform linear arrays characterized by closed form expressions for Hertzian dipoles and detailed finite-difference time-domain (FDTD) simulations of half-wave dipoles illustrate that capacity gains are possible when correlation stems from directional bias in the channel but not when it arises due to compact element spacing.
\end{abstract}

Index Terms-MIMO systems, mutual coupling, antenna arrays, fading channels, information rates, array signal processing.

\section{INTRODUCTION}

$\mathbf{I}$ $\mathrm{T}$ has been established that multiple-input multiple-output (MIMO) architectures can potentially enhance communication spectral efficiency in multipath wireless channels [1]-[3]. However, in a rich multipath environment characterized by wavelength-scale fast fading, the rapid channel variation for highly mobile nodes limits the quality of obtainable channel state information (CSI), which in turn reduces the channel capacity [4]-[6]. In fact, for an i.i.d. Gaussian channel which remains constant over a block of length $T$, the capacity versus the number of transmit antennas achieves a maximum for $T$ antennas [7], in contrast to the results for a constant channel.

While the multipath channel response varies rapidly with position, physical reasoning, measurements, and ray-tracing simulations suggest a slower rate of change of the multipath angular characteristics [6], [8]. Since the channel covariance

Manuscript received December 12, 2006; accepted October 25, 2007. The associate editor coordinating the review of this paper and approving it for publication was R. M. Buehrer. This work was supported in part by the National Science Foundation under Information Technology Grant CCF0428004, and by the U.S. Army Research Office under the Multi-University Research Initiative (MURI) grant W911NF-04-1-0224.

J. W. Wallace is with the School of Engineering and Science, Jacobs University Bremen, 28759 Bremen, Germany (e-mail: wall@ieee.org).

M. A. Jensen is with the Department of Electrical and Computer Engineering, Brigham Young University, Provo, UT, USA 84602 (e-mail jensen@ee.byu.edu).

Digital Object Identifier 10.1109/TWC.2008.05978 is a function of these slowly varying multipath characteristics, communication strategies based on this channel covariance information may be suitable for mobile nodes [5], [9]. Recent work investigating the effect of separable (or Kronecker) correlation on the capacity of block-fading MIMO channels, where the channel covariance is known at transmitter and receiver [10], has proven that (1) adding correlated transmit antennas always increases capacity, (2) optimal signaling involves unitary communications along the principal eigenvectors of the transmit covariance, and (3) for rapidly fading channels $(T=1)$ transmit antenna spacing should be as small possible.

The purpose of this paper is to apply electromagnetic considerations to the analysis in [10], revealing the mechanisms leading to capacity increase with transmit correlation. Unlike previous analyses that constrain the sum of the squares of the transmit signals, we account for the radiated power of the transmit array and explore capacity behavior as a function of the number of correlated antennas as well as their optimal placement using directional channel descriptions with varying levels of multipath. The results of this analysis reveal the impact of antenna coupling and spatial multipath characteristics on the capacity behavior as correlated transmit elements are added to the array. Furthermore, whereas previous work suggests that antennas should be placed arbitrarily close for rapid fading, this new analysis shows that optimal antenna separation is close to that used for conventional phased arrays (0.3 to 0.6 wavelengths).

\section{BACKGROUND}

This section provides introductory material on the channel and scattering models as well as a discussion of power constraints.

\section{A. Channel Model}

As in [7] and [10], we adopt the block-fading channel model

$$
\mathbf{X}=\sqrt{\frac{\rho}{P}} \mathbf{S H}+\mathbf{W}
$$

where $\mathbf{S}$ is the $T \times M$ matrix of complex baseband transmit signals, $\mathbf{X}$ is the $T \times N$ matrix of receive samples, $\mathbf{H}$ is the $M \times N$ channel transfer matrix which is assumed constant over the block of $T$ symbols, and $M$ and $N$ are the number of transmit and receive antennas, respectively. The quantities $P$ and $\rho$ represent the average power generated per unit time by the transmit signal matrix $\mathbf{S}$ and the average signal-tonoise ratio (SNR), respectively. The $T \times N$ matrix $\mathbf{W}$ of 
noise samples consists of i.i.d. elements $W_{i j} \sim \mathcal{C N}(0,1)$, with $\mathcal{C N}\left(\mu, \sigma^{2}\right)$ denoting the univariate complex Gaussian distribution with mean $\mu$ and variance $\sigma^{2}$. We will assume that the transmit signals represent currents, although an analogous formulation could be performed for transmit voltages as well.

The channel matrix $\mathbf{H}$ can be decomposed according to

$$
\mathbf{H}=\mathbf{R}_{T}^{1 / 2} \mathbf{H}_{w} \mathbf{R}_{R}^{1 / 2},
$$

where the i.i.d. elements of $\mathbf{H}_{w}$ are $H_{w, i j} \sim \mathcal{C N}(0,1)$ and $\mathbf{R}_{T}$ and $\mathbf{R}_{R}$ represent the transmit and receive spatial channel covariance matrices, respectively. These covariances are computed from

$$
\begin{aligned}
& \mathbf{R}_{T}=(1 / N) \mathrm{E}\left\{\mathbf{H H}^{H}\right\}, \\
& \mathbf{R}_{R}=(1 / M) \mathrm{E}\left\{\mathbf{H}^{H} \mathbf{H}\right\},
\end{aligned}
$$

where $\mathrm{E}\{\cdot\}$ and $\{\cdot\}^{H}$ represent expectation and conjugate transpose, respectively. The symmetric form of matrix powers for Hermitian matrices $\mathbf{M}^{p}=\boldsymbol{\xi}_{M} \Lambda_{M}^{p} \boldsymbol{\xi}_{M}^{H}$ is always assumed, where $\boldsymbol{\xi}_{M}$ and $\boldsymbol{\Lambda}_{M}$ are the matrix of eigenvectors and the diagonal matrix of eigenvalues of $\mathbf{M}$, respectively. To ensure unit average single-input single-output (SISO) gain, or $[1 /(M N)] \mathrm{E}\left\{\|\mathbf{H}\|_{F}^{2}\right\}=1$ where $\|\cdot\|_{F}$ is the Frobenius norm, $\mathbf{R}_{T}$ and $\mathbf{R}_{R}$ must be scaled such that $\operatorname{Tr}\left\{\mathbf{R}_{T}\right\}=M$ and $\operatorname{Tr}\left\{\mathbf{R}_{R}\right\}=N$, where $\operatorname{Tr}\{\cdot\}$ represents the trace. This model is equivalent to the Kronecker correlation model [11]. We also note that while $\mathbf{H}$ remains constant over a block, there is no temporal correlation of the elements between blocks.

\section{B. Relevant Results on Correlated Block-Fading Channels}

Since many of the results and observations from [10] are studied in this new analysis, we summarize the relevant considerations along with the specific source of the observation within [10]:

$\S 1$ The conditional probability density function (pdf) $p(\mathbf{X} \mid \mathbf{S})$ depends on the transmitted signals $\mathbf{S}$ only through the $T \times T$ matrix $\mathbf{S R}_{T} \mathbf{S}^{H}$. (Source: Property 1 of the conditional pdf.)

$\S 2$ Due to $\S 1$, for a block interval of $T$ and $M>T$, capacity only depends on the $T$ largest eigenvalues of $\mathbf{R}_{T}$. Also, the capacity achieving signal directs transmit power along the $T$ largest eigenvectors of $\mathbf{R}_{T}$. (Source: Lemma 2.)

$\S 3$ Adding a transmit antenna corresponds to augmenting the transmit covariance by a row and column, and this operation almost surely increases the principal eigenvalue of the matrix. (Source: Theorem 2.)

$\S 4$ For correlated transmitters, a signal reassignment strategy is formulated such that mutual information with additional elements remains constant, even though transmit power is reduced. Thus, capacity almost surely increases with additional correlated transmitters. (Source: Theorem 2.)

$\S 5$ For very rapid fading $(T=1)$ and perfect correlation $\left(R_{T, m p}=1\right)$, the principal eigenvalue of $\mathbf{R}_{T}$ is $M$. Thus, for $T=1$, antennas should be placed very close together. (Source: Discussion in Section V-B.)

In what follows, these are referred to as $\S 1-\S 5$.

\section{Mutual Coupling}

To avoid later confusion, we note that the term "mutual coupling" (or "antenna coupling") used in this paper is slightly different from the standard IEEE definition [12], since we are comparing models that discount and include mutual coupling. In [12], the mutual-coupling effect is a change in the pattern and input impedance of a single antenna element when the other antennas are driven (or loaded) as opposed to opencircuited. This change stems from the non-diagonal antenna impedance matrix, meaning that signals couple from one antenna port to another. In this paper, uncoupled antennas refer to elements in an array where the patterns and input impedance are identical to a single or isolated antenna (the impedance matrix is diagonal). For coupled antennas, the impedance matrix is not diagonal, and the patterns may vary from the isolated element pattern as well. Note that this new notion of mutual coupling is basically equivalent to [12], since the current on open-circuited elements will be relatively small, leading to patterns and input impedance that are very similar to isolated elements.

\section{Power Constraints}

In traditional MIMO analyses, the transmit signal is constrained to have unit average power for each antenna and symbol time or

$$
P=P_{\mathrm{tr}}=(1 / T) \mathrm{ETr}\left\{\mathbf{S S}^{H}\right\}=M .
$$

From an electromagnetic standpoint, this is equivalent to constraining the sum of the squared currents on the antenna elements, which for uncoupled antennas also constrains the radiated power. For a lossless transmit array with input impedance matrix $\mathbf{Z}$ and if $\mathbf{s}_{i}$ represents the $i$ th row of the matrix $\mathbf{S}$ of currents, the radiated power for the $i$ th symbol time is [13]

$$
\begin{aligned}
P_{i}=\operatorname{Re}\left\{\mathbf{s}_{i} \mathbf{Z} \mathbf{s}_{i}^{H}\right\} & =\frac{1}{2}\left\{\mathbf{s}_{i} \mathbf{Z} \mathbf{s}_{i}^{H}+\mathbf{s}_{i}^{*} \mathbf{Z}^{*} \mathbf{s}_{i}^{T}\right\} \\
& =\frac{1}{2} \mathbf{s}_{i}\left\{\mathbf{Z}+\mathbf{Z}^{H}\right\} \mathbf{s}_{i}^{H}, \\
& =\mathbf{s}_{i} \operatorname{Re}\{\mathbf{Z}\} \mathbf{s}_{i}^{H},
\end{aligned}
$$

where $\{\cdot\}^{T}$ and $\{\cdot\}^{*}$ represent transpose and conjugate respectively and $\mathbf{A}=\operatorname{Re}\{\mathbf{Z}\}$ is referred to as the coupling matrix. In this formulation, we have taken the transpose of the second (scalar) term on the right-hand side of (6) and have used that $\mathbf{Z}$ is symmetric for reciprocal antennas. The radiated power averaged over the block can be expressed as

$$
P=P_{\mathrm{rad}}=(1 / T) \mathrm{ETr}\left\{\mathbf{S} \mathbf{A} \mathbf{S}^{H}\right\} .
$$

The coupling matrix A can also be computed based on the element radiation patterns. If $\Omega$ denotes a solid angle coordinate and $f_{m}(\Omega)$ represents the radiation pattern for the $m$ th antenna in response to unit current with all other elements terminated in an open-circuit, then we can write

$$
A_{m p}=\int_{\Omega_{0}} d \Omega f_{m}(\Omega) f_{p}^{*}(\Omega),
$$

where the region of integration $\Omega_{0}$ is generally a full $4 \pi$ steradians. We note, however, that for instructional purposes 
we may limit $\Omega_{0}$ to a circle in the azimuthal plane. The opencircuit termination for radiation pattern specification stems from the fact that the transmitted signals are considered currents, so that the total radiated field distribution may be computed as the superposition of the patterns weighted by the currents.

To allow for fair comparison between results for different array types, impedance matrices will be divided by the real part of the impedance for an isolated element of the same type. Similarly, radiation patterns will be divided by the quantity

$$
\left[\int_{\Omega_{0}} d \Omega|\hat{f}(\Omega)|^{2}\right]^{1 / 2}
$$

where $\hat{f}(\Omega)$ is the radiation pattern of an isolated element of the same type.

We will focus on ideal Hertzian (infinitesimal) [14] or halfwave dipoles oriented perpendicular to the azimuthal plane and arranged in a uniform linear array (ULA) with interelement spacing $\Delta x$ in wavelengths. The Hertzian dipole array is interesting because it exhibits coupling in the sense that $\mathbf{A}$ is in general full, but the introduction of an open-circuited element next to a driven element will not change the driven element radiation pattern. This behavior of the pattern ensures that addition of an element will not change the entries in $\mathbf{R}_{T}$ corresponding to existing elements, a prerequisite behavior for $\S 4$ to hold. The half-wave dipoles are used as a more practical example of an antenna for which this pattern behavior is not observed.

The coupling matrix $\mathbf{A}$ for each array must be constructed from an electromagnetic network description obtained by measurement, analysis, or simulation [15]. For Hertzian dipoles, we will assume a two-dimensional (2D) scenario $\left(\Omega_{0}\right.$ represents a circle in the azimuthal plane in (10)) so that the results highlight the impact of the array geometry. The resulting coupling matrix has elements

$$
A_{m p}=J_{0}[2 \pi \Delta x(m-p)],
$$

where $J_{0}(\cdot)$ is the zeroth order Bessel function. For halfwave dipole arrays of up to four elements, the patterns and impedance matrix are computed using detailed finitedifference time-domain (FDTD) simulations [16], [17]. The antenna geometry, simulation strategy, and FDTD parameters are identical to those described in [13].

In the analysis that follows, we construct $\mathbf{S}$ using the power constraint in (5), but then interpret the results in the context of the power radiated by the transmit array. This approach is suboptimal, since the optimal solution would find $\mathbf{S}$ by directly constraining the radiated power. Nonetheless, the suboptimal scheme is convenient for several reasons:

1. the traditional power constraint avoids impractical supergain solutions [18],

2. since the signaling strategy is the same as in $\S 2$, the results of this analysis can be directly compared to those from the prior analysis,

3. realistic systems with no detailed knowledge of A may implement such a method to ensure the radiated power satisfies regulatory constraints, and

4. the analysis is greatly simplified since the eigenstructure of $\mathbf{A}$ is not required.

\section{E. Directional Scattering Model}

Studying capacity behavior for correlated block-fading MIMO systems requires realistic covariance matrix representations. The symbols used in the following can represent quantities at either transmit or receive. This work employs a 2D directional channel model, where the electric field $e_{s}(\phi)$ departing from the transmit array or incident on the receive array is uncorrelated in angle, resulting in $\mathrm{E}\left\{e_{s}(\phi) e_{s}^{*}\left(\phi^{\prime}\right)\right\}=$ $p(\phi) \delta\left(\phi-\phi^{\prime}\right)$, where $p(\phi)$ is the power angular spectrum (PAS) at angle $\phi$ in the azimuthal plane. The spatial covariance matrix $\mathbf{R}_{T}$ or $\mathbf{R}_{R}$ has elements computed from the integral [13]

$$
R_{m p}=\int_{0}^{2 \pi} d \phi f_{m}(\phi) f_{p}^{*}(\phi) p(\phi) .
$$

For a ULA of Hertzian dipoles, $f_{m}(\phi)=\exp [j 2 \pi m \Delta x \cos \phi]$.

To study the impact of channel propagation conditions on performance, we will consider three different forms of $p(\phi)$. The first case of full angular spread models an environment with rich scattering. Using $p(\phi)=1 /(2 \pi)$ in (13) with Hertzian dipole patterns leads to the classical model $R_{m p}=$ $J_{0}[2 \pi \Delta x(m-p)]$ found in [19].

In the second case, discrete multipath components are assumed, leading to a PAS of the form

$$
p(\phi)=\sum_{\ell=1}^{L} \beta_{\ell} \delta\left(\phi-\phi_{\ell}\right),
$$

where $L$ is the number of paths, $\beta_{\ell}$ and $\phi_{\ell}$ are the power and angle of the $\ell$ th multipath, and $\delta(\cdot)$ is the Dirac delta function. This leads to

$$
R_{m p}=\sum_{\ell=1}^{L} \beta_{\ell} f_{m}\left(\phi_{\ell}\right) f_{p}^{*}\left(\phi_{\ell}\right)
$$

In the final case, a single cluster of multipath arrivals or departures at a mean angle of $\bar{\phi}$ has a PAS described by the von Mises distribution [20], or

$$
p(\phi)=\frac{\exp [\kappa \cos (\phi-\bar{\phi})]}{2 \pi I_{0}(\kappa)},
$$

where $\kappa$ is the directivity parameter and $I_{0}(\cdot)$ is the zeroth order modified Bessel function. The $3 \mathrm{~dB}$ width of the multipath cluster is given by $2 \cos ^{-1}\left[1+\kappa^{-1} \ln (0.5)\right]$ or approximately $135^{\circ} / \sqrt{\kappa}$ for $\kappa \geq 1$.

\section{CAPACity Growth With TRansmit Antennas}

Result $\S 4$ indicates guaranteed capacity growth with the addition of correlated transmit antennas. Our purpose here is to incorporate radiated power considerations into the analysis to see if the guaranteed capacity growth is still applicable for close antenna spacing.

\section{A. Eigenbeamforming Interpretation of the Channel}

Understanding the capacity behavior for correlated blockfading channels is facilitated by an eigenbeamforming interpretation. Substituting (2) into (1) and taking the eigenvalue 
decomposition (EVD) of the covariance matrices yields

$$
\begin{aligned}
\mathbf{X} & =\sqrt{\frac{\rho}{P}} \mathbf{S R}_{T}^{1 / 2} \mathbf{H}_{w} \mathbf{R}_{R}^{1 / 2}+\mathbf{W}, \quad \text { or } \\
\underbrace{\mathbf{X} \boldsymbol{\xi}_{R}}_{\mathbf{X}^{\prime}} & =\sqrt{\frac{\rho}{P}} \underbrace{\mathbf{S} \boldsymbol{\xi}_{T}}_{\mathbf{S}^{\prime}} \boldsymbol{\Lambda}_{T}^{1 / 2} \underbrace{\boldsymbol{\xi}_{T}^{H} \mathbf{H}_{w} \boldsymbol{\xi}_{R}}_{\mathbf{H}_{w}^{\prime}} \boldsymbol{\Lambda}_{R}^{1 / 2} \underbrace{\boldsymbol{\xi}_{R}^{H} \boldsymbol{\xi}_{R}}_{\mathbf{I}}+\underbrace{\mathbf{W} \boldsymbol{\xi}_{R}}_{\mathbf{W}^{\prime}} .
\end{aligned}
$$

The unitary transformations do not change the statistics of the channel and noise $\left(\mathbf{H}_{w}^{\prime}\right.$ has the same statistics as $\mathbf{H}_{w}$ and $\mathbf{W}^{\prime}$ has the same statistics as $\mathbf{W}$ ) nor the capacity [7]. Therefore, we will continue to use the unprimed representations for these two quantities in the following to simplify notation, or

$$
\mathbf{X}^{\prime}=\sqrt{\frac{\rho}{P}} \mathbf{S}^{\prime} \boldsymbol{\Lambda}_{T}^{1 / 2} \mathbf{H}_{w} \boldsymbol{\Lambda}_{R}^{1 / 2}+\mathbf{W} .
$$

The behavior of the channel is depicted graphically in Figure 1.

This formulation reveals that communication along the eigenvectors of the transmit and receive covariance (eigenbeamforming) transforms the channel such that the virtual transmit and receive elements observe an i.i.d. channel. However, each virtual element has a different gain due to the scaling by the covariance eigenvalues so that the effective channel has independent but not identically distributed elements.

\section{B. Effective Gain}

Consider a system with $M$ transmit antennas to which $\Delta M$ additional antennas are introduced. Let $(\cdot)$ represent an abstract quantity (such as $\mathbf{R}_{T}, \mathbf{S}$, etc.) for the original system and (.) represent the same quantity for the system with additional elements. Consistent with $\S 4$ and since mutual information depends on the distribution of $\mathbf{S R}_{T} \mathbf{S}^{H}=\mathbf{S}^{\prime} \boldsymbol{\Lambda}_{T} \mathbf{S}^{\prime H}$, we can ensure that our two systems have the same mutual information if

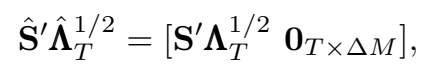

where $\mathbf{0}_{T \times \Delta M}$ is a $T \times \Delta M$ matrix of zeros. This leads to

$$
\hat{\mathbf{S}}^{\prime}=\left[\mathbf{S}^{\prime} \boldsymbol{\Lambda}_{T}^{1 / 2}\left(\hat{\mathbf{\Lambda}}_{T}^{-1 / 2}\right)_{M} \mathbf{0}_{T \times \Delta M}\right],
$$

where $\left(\hat{\boldsymbol{\Lambda}}_{T}^{-1 / 2}\right)_{M}$ indicates the upper left $M \times M$ sub-block of $\hat{\boldsymbol{\Lambda}}_{T}^{-1 / 2}$.

If the radiation patterns of the original $M$ antennas do not change with the addition of the $\Delta M$ elements, then $\S 3$ and $\S 4$ will hold and the power radiated by the system with $M+\Delta M$ antennas will be lower than that for the original system. Note that this behavior is not guaranteed if the element patterns change with the introduction of the additional elements, which motivates the use of FDTD-characterized half-wave dipoles in this study [13], [21]. We note, however, that even for the half-wave dipoles, the fact that patterns are computed with all other elements terminated in an open-circuit will minimize this pattern distortion.

To quantify the performance gain enabled by the addition of $\Delta M$ antennas, we define the effective gain as the ratio of the power radiated by the original system to that radiated by the augmented system, or

$$
G_{\text {eff }}=\frac{\operatorname{ETr}\left\{\mathbf{S} \mathbf{A} \mathbf{S}^{H}\right\}}{\operatorname{ETr}\left\{\hat{\mathbf{S}} \hat{\mathbf{A}} \hat{\mathbf{S}}^{H}\right\}} .
$$

Thus, if adding the $\Delta M$ antennas decreases radiated power, $G_{\text {eff }}>1$, which is equivalent to capacity growth since scaling the power so that both systems achieve the same radiated power will boost the mutual information for the augmented system.

\section{Computational Results}

In this section, the effective gain metric (22) is applied to the propagation environments introduced in Section IIE. An analytical treatment of the Hertzian dipoles reveals the general system behavior in each case, while examples using half-wave dipoles demonstrate that the general observations are relevant for more practical geometries. Because the propagation environment significantly impacts the results and an exhaustive treatment is not possible, we consider four special cases that are simple, yet together approximate most propagation environments of practical interest.

1) Full Angular Spread: First, consider the case of full angular spread with transmit covariance $R_{T, m p}=J_{0}[2 \pi \Delta x(m-$ $p)$ ]. Since in this circumstance $\mathbf{R}_{T}=\mathbf{A}$, the radiated power for $M$ antennas is simply

$$
P_{\text {rad }}=\frac{1}{T} \mathrm{E} \operatorname{Tr}\left\{\mathbf{S} \mathbf{A} \mathbf{S}^{H}\right\}=\frac{1}{T} \mathrm{E} \operatorname{Tr}\left\{\mathbf{S}^{\prime} \boldsymbol{\Lambda}_{T} \mathbf{S}^{\prime H}\right\} .
$$

Therefore, if the scheme outlined in Section III-B is used to maintain constant mutual information as antennas are added, the radiated power will also remain constant so that $G_{\text {eff }}=1$. This result is intuitive since for uniform departures, spatial filtering should not change the system performance. This case highlights one of the key features of systems with mutual coupling: changing the transmit antenna configuration can only enhance beamforming gain if the increase in correlation is not offset by increased coupling.

2) Single Departure: Next consider the case where propagation to the receiver occurs for only a single departure direction $\phi$ with $L=1$ and $\beta_{1}=1$ in (14). In this case, a single spatial transmission mode exists with eigenvalue $\lambda=M$ and eigenvector with elements

$$
v_{m}=1 / \sqrt{M} \exp (j 2 \pi m \Delta x \cos \phi) .
$$

Optimal transmission involves exciting this mode with $\mathbf{S}=$ $\mathbf{s}^{\prime} \mathbf{v}^{H}$, where $\mathbf{s}^{\prime}$ is the $T \times 1$ vector of time symbols for the current block, producing a radiated power of

$$
\begin{aligned}
P_{\mathrm{rad}} & =(1 / T) \mathrm{E} \operatorname{Tr}\left\{\mathbf{S A} \mathbf{S}^{H}\right\} \\
& =\underbrace{(1 / T) \mathrm{E}\left\{\mathbf{s}^{\prime H} \mathbf{s}^{\prime}\right\}}_{=1} \operatorname{Tr}\left\{\mathbf{v}^{H} \mathbf{A} \mathbf{v}\right\} \lambda^{-1} .
\end{aligned}
$$

For Hertzian dipoles, this yields

$$
\begin{aligned}
& \operatorname{Tr}\left\{\mathbf{v}^{H} \mathbf{A v}\right\} \\
& =\frac{1}{M} \sum_{m=1}^{M} \sum_{p=1}^{M} \exp [-j 2 \pi \Delta x(m-p) \cos \phi] J_{0}[2 \pi \Delta x(m-p)] \\
& =1+\frac{1}{M} \sum_{m=1}^{M-1}(M-m) \cos (2 \pi m \Delta x \cos \phi) J_{0}(2 \pi m \Delta x) .
\end{aligned}
$$

The effective gain of an $M$ element system over the single antenna system is $G_{\text {eff }}=M / \operatorname{Tr}\left\{\mathbf{v}^{H} \mathbf{A v}\right\}$. Although this gain 


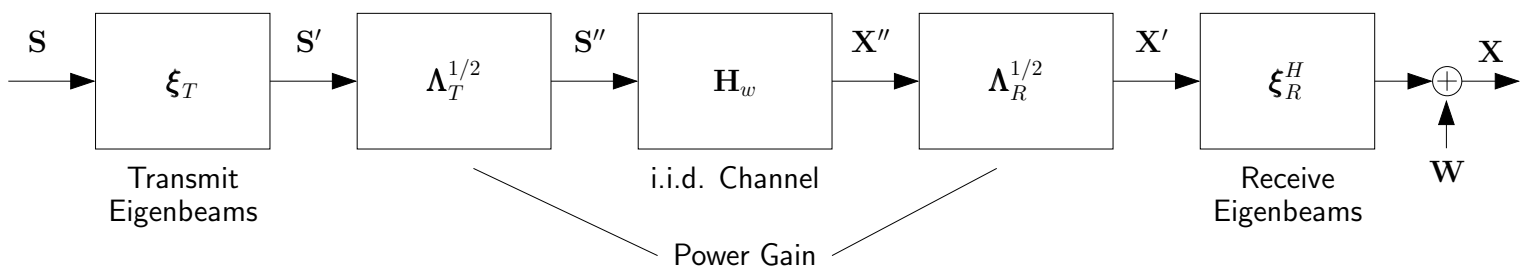

Fig. 1. Graphical representation of channels with separable (Kronecker) correlation

may be smaller or larger than $M$ depending on $\mathbf{A}$ and $\mathbf{v}$, it will scale linearly with $M$ provided that the antenna elements are spaced sufficiently far apart.

3) Two Departures: For two departure paths $\phi_{1}$ and $\phi_{2}$ with gains $\beta_{1}$ and $\beta_{2}$ in (14), the eigenvectors and eigenvalues of $\mathbf{R}_{T}$ may again be found in closed form. The two eigenvalues are

$$
\begin{aligned}
\lambda_{1,2} & =\frac{M}{2}\left[1 \pm \sqrt{\left(\beta_{1}-\beta_{2}\right)^{2}+\frac{4|\Gamma|^{2}}{M^{2}} \beta_{1} \beta_{2}}\right] \\
\Gamma & =e^{-j \epsilon} \frac{\sin \left[\pi \Delta x M\left(\cos \phi_{1}-\cos \phi_{2}\right)\right]}{\sin \left[\pi \Delta x\left(\cos \phi_{1}-\cos \phi_{2}\right)\right]},
\end{aligned}
$$

where $\epsilon=\pi \Delta x(M-1)\left(\cos \phi_{1}+\cos \phi_{2}\right)$. The $m$ th element of the eigenvector $\mathbf{v}_{k}$ for eigenvalue $\lambda_{k}, k \in\{1,2\}$ is

$$
\left(\mathbf{v}_{k}\right)_{m}=\alpha_{k 1} e^{j 2 \pi m \Delta x \cos \phi_{1}}+\alpha_{k 2} e^{j 2 \pi m \Delta x \cos \phi_{2}}
$$

where $\alpha_{k 2} / \alpha_{k 1}=\left(\lambda_{k}-\beta_{1} M\right) /\left(\beta_{1} \Gamma\right)$. For simplicity, we will assume equal path gains $\left(\beta_{1}=\beta_{2}=1 / 2\right)$ which leads to

$$
\begin{aligned}
& \lambda_{1,2}=\frac{M}{2}\left(1 \pm \frac{|\Gamma|}{M}\right), \\
& \frac{\alpha_{k 2}}{\alpha_{k 1}}= \pm \frac{|\Gamma|}{\Gamma}= \pm \exp (-j \angle \Gamma),
\end{aligned}
$$

where $L$. takes the phasor angle of the complex argument. This produces $\lim _{M \rightarrow \infty}|\Gamma| / M=0$, so that $\lambda_{1}$ and $\lambda_{2}$ each approach $M / 2$. Physically, this fact means that a large array can place independent beams in the $\phi_{1}$ and $\phi_{2}$ directions, and the two-ray model simply becomes two uncoupled one-ray models with half of the original gain.

To explore the case of finite $M$, consider a system with $M=2$ transmit elements and signal block $\mathbf{S}$. Increasing the number of antennas to $M=2+\Delta M$ and using the strategy in Section III-B yields a radiated power of

$$
\begin{aligned}
P_{\text {rad }} & =(1 / T) \operatorname{ETr}\left\{\hat{\mathbf{S}} \hat{\mathbf{A}} \hat{\mathbf{S}}^{H}\right\} \\
& =(1 / T) \operatorname{Tr}\left\{\mathrm{E}\left\{\mathbf{S}^{\prime H} \mathbf{S}^{\prime}\right\} \boldsymbol{\Lambda}_{T}^{1 / 2} \hat{\boldsymbol{\Lambda}}_{T, 2}^{-1 / 2} \hat{\mathbf{V}}^{H} \hat{\mathbf{A}} \hat{\mathbf{V}} \hat{\mathbf{\Lambda}}_{T, 2}^{-1 / 2} \boldsymbol{\Lambda}_{T}^{1 / 2}\right\} \\
& \stackrel{(\text { a) }}{\approx} \operatorname{Tr}\left\{\boldsymbol{\Lambda}_{T} \hat{\mathbf{\Lambda}}_{T, 2}^{-1} \hat{\mathbf{V}}^{H} \hat{\mathbf{A}} \hat{\mathbf{V}}\right\} \\
& =\lambda_{1} \hat{\lambda}_{1}^{-1} \hat{\mathbf{v}}_{1}^{H} \hat{\mathbf{A}} \hat{\mathbf{v}}_{1}+\lambda_{2} \hat{\lambda}_{2}^{-1} \hat{\mathbf{v}}_{2}^{H} \hat{\mathbf{A}}_{\mathbf{v}},
\end{aligned}
$$

where approximation (a) comes from assuming $T \rightarrow \infty$ and $\hat{\mathbf{V}}=\left[\begin{array}{ll}\hat{\mathbf{v}}_{1} & \hat{\mathbf{v}}_{2}\end{array}\right]$ contains the two eigenvectors of $\hat{\mathbf{R}}_{T}$. The effective gain becomes

$$
G_{\text {eff }}=\frac{\mathbf{v}_{1}^{H} \mathbf{A} \mathbf{v}_{1}+\mathbf{v}_{2}^{H} \mathbf{A} \mathbf{v}_{2}}{\lambda_{1} \hat{\lambda}_{1}^{-1} \hat{\mathbf{v}}_{1}^{H} \hat{\mathbf{A}} \hat{\mathbf{v}}_{1}+\lambda_{2} \hat{\lambda}_{2}^{-1} \hat{\mathbf{v}}_{2}^{H} \hat{\mathbf{A}} \hat{\mathbf{v}}_{2}} .
$$

The products $\lambda_{k} \hat{\lambda}_{k}^{-1}$ indicate that effective gain mainly results from an increase in the eigenvalues which is a result of

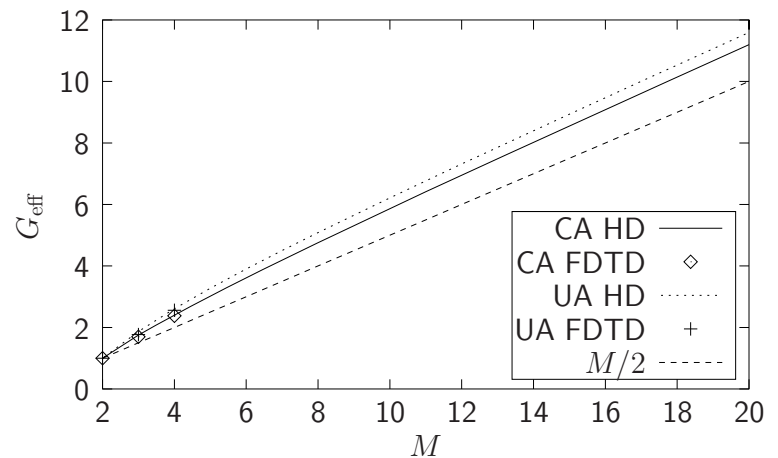

Fig. 2. Mean effective gain for the two-path model with $\Delta x=0.5$ from Monte Carlo simulations for coupled (CA) and uncoupled antennas (UA) for either Hertzian dipoles (HD) or FDTD simulations of half-wave dipoles (FDTD)

improved beamforming. If antennas are placed sufficiently far apart, $\mathbf{A} \approx \mathbf{I}$, and the effective gain becomes

$$
G_{\text {eff }}=\frac{2}{\lambda_{1} \hat{\lambda}_{1}^{-1}+\lambda_{2} \hat{\lambda}_{2}^{-1}} .
$$

Figure 2 plots the effective gain as a function of $M$ for the two-ray equal-gain model with $\Delta x=0.5$ averaged over $10^{4}$ random realizations with $\phi_{1}$ and $\phi_{2}$ uniformly distributed on $[0,2 \pi]$. Including the coupling using (33) yields a capacity growth almost identical to that of the uncoupled case (34), and only a few elements are required to approach the asymptotic growth rate of $M / 2$. Results for the half-wave dipoles are also shown up through $M=4$. The values for the two antenna types are nearly indistinguishable, indicating that for this geometry the key behavior is well captured by the simple Hertzian dipole model.

4) Single Cluster Model: Finally, consider the case of a single continuous cluster of departures described by the von Mises distribution with PAS (16) and corresponding transmit covariance

$$
R_{T, m p}=I_{0}\left(\sqrt{\kappa^{2}-y_{m p}^{2}+j 2 \pi \kappa y_{m p} \cos \bar{\phi}}\right) / I_{0}(\kappa),
$$

where $y_{m p}=2 \pi(m-p) \Delta x$. Assuming communication on just the single dominant spatial mode, the effective gain is

$$
G_{\text {eff }}=\frac{\mathbf{v}_{1}^{H} \mathbf{A} \mathbf{v}_{1}}{\lambda_{1} \hat{\lambda}_{1}^{-1} \hat{\mathbf{v}}_{1}^{H} \hat{\mathbf{A}} \hat{\mathbf{v}}_{1}} .
$$

Figures 3 and 4 plot the effective gain for uncoupled and coupled antennas, respectively, where a single cluster departs in the endfire direction $(\bar{\phi}=0)$, antenna spacing is $\Delta x=0.5$ wavelengths, and various values of $\kappa$ are considered. For uncoupled antennas, the capacity growth is directly proportional 


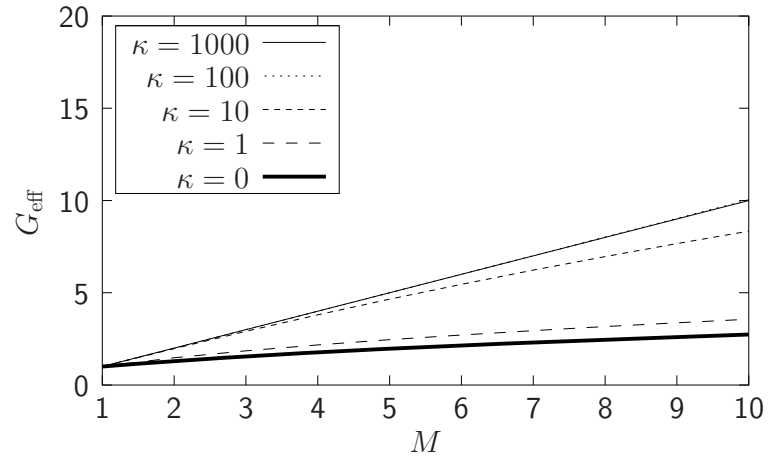

Fig. 3. Effective gain versus the number of antenna elements for a single endfire cluster for uncoupled antennas

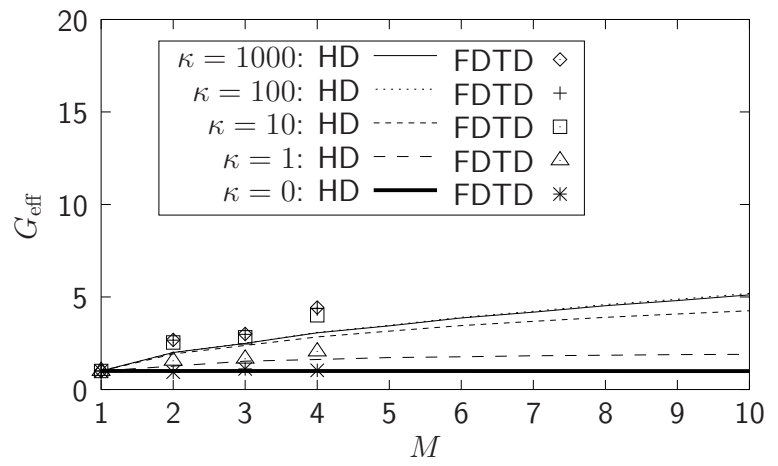

Fig. 4. Effective gain versus the number of antenna elements for a single endfire cluster with coupled antennas, consisting of either Hertzian dipoles (HD) or half-wave dipoles (FDTD)

to $\kappa$ (inversely proportional to angular spread), and for $\kappa \rightarrow \infty$ effective gain approaches $M$ just as in the case of a single departure. For coupled antennas, the case $\kappa=0$ (equivalent to full angular spread) has no effective gain. The behavior is slightly different for the two types of coupled antennas. The gain of Hertzian dipoles is inhibited due to the increased radiated power associated with endfire excitation at $\Delta x=0.5$. On the other hand, the half-wave dipoles simulated with FDTD have gain close to the uncoupled case, likely due to the fact that the coupled radiation patterns for endfire excitation tend to confine power more to the azimuthal plane, thus offsetting the effect seen with Hertzian dipoles.

While results for a cluster at broadside are not shown for the sake of brevity, we note that the Hertzian and halfwave dipoles have almost identical performance for this case. Furthermore, the effective gain for the coupled elements is actually higher than that for the uncoupled elements at $\Delta x=$ 0.5. The key conclusion from all of these results is the confirmation that the effective gain can grow linearly with the number of elements provided that the channel is characterized by directional bias, but that coupling can degrade or enhance the performance.

\section{Antenna Placement for Rapidly FADing CHANNELS}

For rapidly fading channels $(T=1), \S 5$ indicates that only one spatial mode should be used and therefore antenna placement should be chosen to maximize the principal channel eigenvalue. Equation (13) indicates that this is accomplished by letting $\Delta x \rightarrow 0$ so that $R_{T, m p} \rightarrow 1$, producing a single nonzero eigenvalue of $M$. This concept of placing antennas as close together as possible is troubling from an electromagnetic perspective, since for $\Delta x=0$ the antennas should function as a single element.

The apparent contradiction arises because the traditional power constraint (5) is not useful for close spacings [13]. For example, for $\Delta x=0$, Hertzian dipoles have $A_{m p}=1$, meaning that excitation along the principal eigenvector leads to $P_{\text {rad }}=M$ (which equals the channel eigenvalue). Although the gain increases by a factor of $M$, the radiated power increases by $M$ as well, and therefore the effective gain is unity. The following analysis studies this issue in more detail.

\section{A. Effective Gain for $M=\Delta M=1$}

For a single transmit antenna and $T=1$, we have $\mathbf{S}^{\prime}=[\alpha]$ where $|\alpha|=1$ and $P_{\text {rad }}=1$. For two transmit antennas, the scheme outlined in Section III-B produces $\hat{\mathbf{S}}=\left(\alpha / \sqrt{\lambda_{1}}\right) \mathbf{v}_{1}^{H}$, where $\lambda_{1}$ is the dominant eigenvalue of $\hat{\mathbf{R}}_{T}$ with corresponding eigenvector $\mathbf{v}_{1}$. The effective gain is therefore $G_{\text {eff }}=$ $\lambda_{1} /\left(\mathbf{v}_{1}^{H} \hat{\mathbf{A}} \mathbf{v}_{1}\right)$. Note that in this case, the generated signal matrix $\hat{\mathbf{S}}$ is optimal since for $T=1$, the optimal beamformer is the dominant eigenvector of $\mathbf{R}_{T}$.

This result can be further simplified analytically for this two-antenna case. The two-antenna transmit covariance matrix is of the form

$$
\mathbf{R}_{T}=\left[\begin{array}{cc}
1 & \gamma \\
\gamma^{*} & 1
\end{array}\right]
$$

with eigenvalues $\lambda_{1,2}=1 \pm|\gamma|$ and eigenvectors

$$
\mathbf{v}_{1,2}=(1 / \sqrt{2})[1 \quad \pm \exp (-j \angle \gamma)]^{T} .
$$

For Hertzian dipoles, we obtain

$$
G_{\text {eff }}=\frac{1+|\gamma|}{1+\cos (\angle \gamma) J_{0}(2 \pi \Delta x)} .
$$

Thus, we are left with finding the antenna spacing that maximizes the effective gain for a given value of $\gamma$.

\section{B. Computational Results}

We will again consider the different propagation environments introduced in Section II-E.

1) Full Angular Spread: The case of full angular spread and Hertzian dipoles is trivial, since $\gamma=J_{0}(2 \pi \Delta x)$, and (39) gives $G_{\text {eff }}=1$, regardless of the antenna spacing. Thus, any increase in correlation due to reduced spacing is exactly offset by an increase in radiated power. To avoid difficulties with element coupling, antenna spacing should be as large as possible.

2) L-path Model: Next we consider the case of $L$ discrete paths, each having a mean power of $1 / L$. The path directions $\phi_{\ell}$ are assumed to be i.i.d. uniform on $[0,2 \pi]$. Figure 5 plots the mean effective gain computed by averaging $G_{\text {eff }}$ over $10^{4}$ channel realizations as a function of spacing. As expected, the effective gain decreases with increasing multipath. Also, antennas should be placed no closer than about 0.4 wavelengths since at this point coupling begins to counteract the benefits of correlation leading to a reduction in $G_{\text {eff }}$. We further observe that the behavior of the two antenna types is very similar, with 


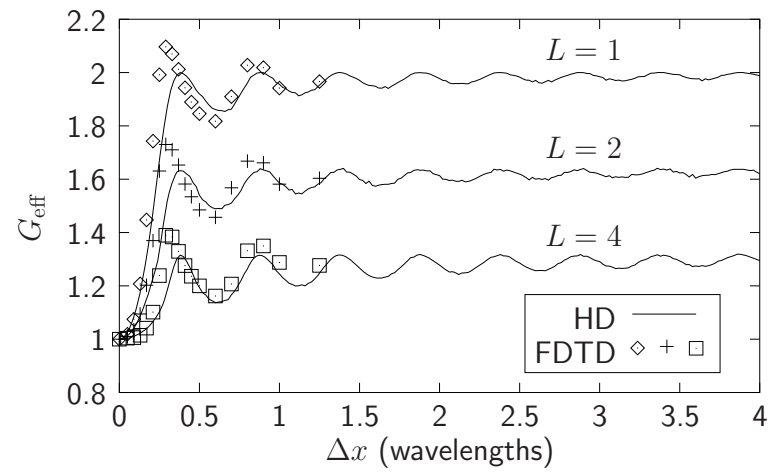

Fig. 5. Effective gain for the $L$-path model with $L=1,2,4$ for Hertzian dipoles (HD) and half-wave dipoles (FDTD)

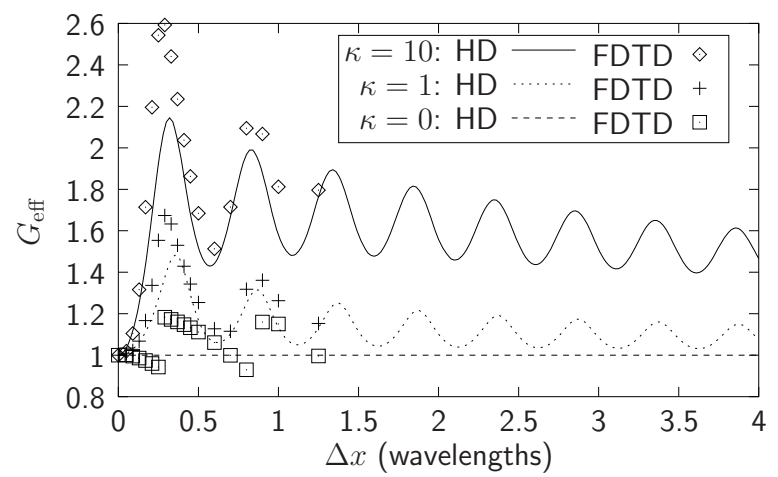

Fig. 6. Effective gain as a function of antenna spacing assuming a single departing cluster distributed according to the von Mises distribution for three values of $\kappa$ with $\bar{\phi}=0$ for Hertzian dipoles (HD) and half-wave dipoles (FDTD)

a possible exception around $\Delta x=0.25$. At this point, the radiation patterns for the half-wave dipoles better confine the radiation to the horizontal plane, providing a slight increase in the effective gain. It is important to emphasize that $\S 5$ suggests $G_{\text {eff }}=2$ as $\Delta x \rightarrow 0$, in contrast to the more physically intuitive behavior observed here.

3) Single Cluster Model: Finally, we consider a single departing cluster described with a von Mises angular distribution, where $\bar{\phi}$ and $\kappa$ are fixed. For a specific array orientation, $\gamma$ is computed from (35) with $y_{12}=-2 \pi \Delta x$. Figures 6 and 7 plot $G_{\text {eff }}$ versus $\Delta x$ for three values of $\kappa$ for endfire $(\bar{\phi}=0)$ and broadside $(\bar{\phi}=\pi / 2)$ mean departure angle, respectively.

Here again, the results reveal that increased multipath (decreased $\kappa$ ) causes a gain reduction. However, in contrast to the results observed for the discrete path model, very large spacings are now less desirable. We have observed that this behavior stems from the fixed mean departure angle, as the effective gain averaged over a uniformly distributed sequence of mean departure angles looks similar to the curves for the discrete path model (where angles of arrival are already uniformly distributed). The key observation from this result is that if array orientation relative to the multipath can be controlled, reasonably close spacings may be advantageous. When the arrival angles are more random, very wide spacings appear to be nearly as optimal as narrow spacings.

Although the Hertzian and half-wave dipoles have similar behavior, there is once again an obvious discrepancy around the point $\Delta x=0.25$. Also, the effective gain for half-wave

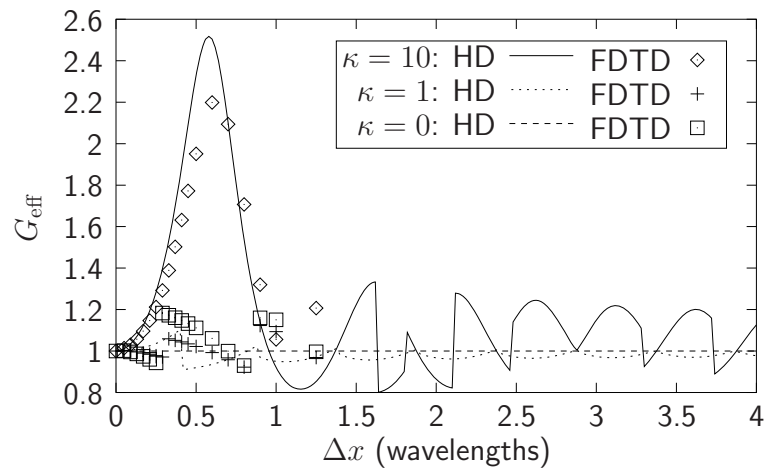

Fig. 7. Effective gain as a function of antenna spacing assuming a single departing cluster distributed according to the von Mises distribution for three values of $\kappa$ with $\bar{\phi}=\pi / 2$ for Hertzian dipoles (HD) and half-wave dipoles (FDTD)

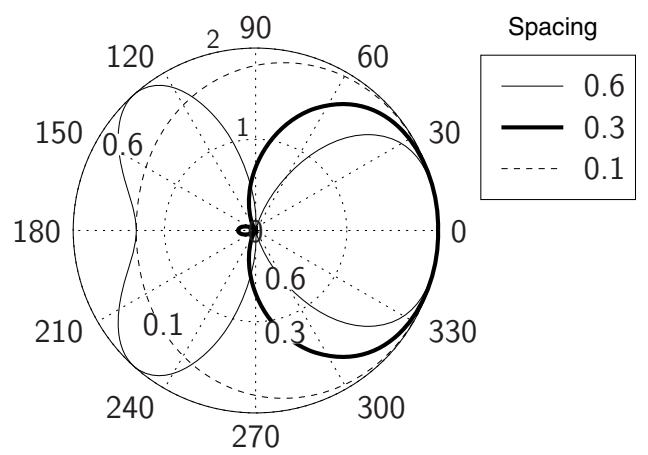

Fig. 8. Radiation patterns for endfire excitation $(\bar{\phi}=0)$ on a 2-element array for antenna spacings $\Delta x=\{0.1,0.3,0.6\}$ (wavelengths)

dipoles is no longer unity for all spacings with full azimuthal spread of the multipath. This is due to the fact that the radiation patterns of the antennas change as a function of spacing, with the patterns at certain spacings increasing the gain in the horizontal plane.

For a realistic cluster size of $14^{\circ}(\kappa=10)$, the optimal spacing for endfire and broadside departures is approximately 0.3 and 0.6 wavelengths, respectively. The optimality of these spacings can be understood by phasing the two antennas such that the main beam is steered in direction $\bar{\phi}$, or $\mathbf{v}^{H}=$ $(1 / \sqrt{2})[1 \exp (j 2 \pi \Delta x \cos \bar{\phi})]$. The resulting radiation pattern of the array is

$$
P(\phi)=\cos ^{2}[\pi \Delta x(\cos \phi-\cos \bar{\phi})],
$$

which is plotted in Figure 8 for $\bar{\phi}=0$ (endfire excitation). The spacing of $\Delta x=0.3$ is near the point at which a single main lobe is created in the endfire direction. Larger spacings create sidelobes that contribute to the radiated power but not to gain through the channel, while smaller spacings create a more uniform pattern with little spatial selectivity. For broadside excitation (not plotted), we have a similar scenario as endfire excitation, except that the beam with good spatial selectivity and no sidelobes is achieved for a spacing near $\Delta x=0.6$ wavelengths.

\section{Single Cluster Model with Input Power Constraint}

One of the strange aspects of the effective gain curves for broadside excitation in Figure 7 is the presence of discontinu- 
ities. These artifacts appear as $\gamma$ (which is purely real for $\bar{\phi}=$ $\pi / 2$ ) changes sign abruptly as the spacing increases. Since the signaling method communicates on the dominant eigenvector in (38), element excitation suddenly changes from from evenmode (same current) to odd-mode (opposite currents). This observation highlights the suboptimality of communicating on the principal eigenvector of $\mathbf{R}_{T}$ and ignoring the effect of antenna coupling in signal construction.

To explore the idea of optimal signaling with covariance information, consider only constraining the signal matrix $\mathbf{S}$ such that $P=P_{\text {rad }}=M$. This constraint can be transformed into the traditional power constraint by making the substitution $\mathbf{S}=\mathbf{S}^{\prime} \mathbf{A}^{-1 / 2}$, and the relationship for the channel becomes

$$
\mathbf{X}=\sqrt{\frac{\rho}{M}} \mathbf{S}^{\prime} \underbrace{\mathbf{A}^{-1 / 2} \mathbf{R}_{T}^{1 / 2}}_{\mathbf{R}_{T}^{\prime 1 / 2}} \mathbf{H}_{w} \mathbf{R}_{R}^{1 / 2}+\mathbf{W},
$$

thus creating the effective transmit covariance

$$
\mathbf{R}_{T}^{\prime}=\mathbf{A}^{-1 / 2} \mathbf{R}_{T} \mathbf{A}^{-(1 / 2) H},
$$

which includes both the effects of antenna correlation and coupling. For $T=1$, the optimal strategy now directs power along the principal eigenvector of $\mathbf{R}_{T}^{\prime}$ rather than $\mathbf{R}_{T}$.

As $\Delta x \rightarrow 0$, this strategy is problematic, since the supergain effect becomes significant [18]. To avoid the appearance of impractical supergain solutions, we assume a modified coupling matrix of the form [22]

$$
\mathbf{A}=\eta \mathbf{A}_{0}+(1-\eta) \mathbf{I}, \quad \mathbf{R}_{T}=\eta \mathbf{R}_{T 0}
$$

where $\mathbf{A}_{0}$ and $\mathbf{R}_{T 0}$ are the radiation-only coupling and covariance matrices defined previously, and $\eta$ is the antenna efficiency. The first and second terms of $\mathbf{A}$ represent radiation and ohmic loss, respectively. The ohmic loss regularizes the inverse of $\mathbf{A}$, which physically corresponds to removal of supergain solutions. Since A now contains loss, the formulation actually constrains the system input power.

Assuming $M=2$, closed-form solutions for $G_{\text {eff }}$ with the input power constraint are possible. The coupling matrix is of the form

$$
\mathbf{A}=\left[\begin{array}{cc}
1 & a \\
a^{*} & 1
\end{array}\right]
$$

with eigenvalues $g_{1,2}=1 \pm|a|$ and eigenvectors $\mathbf{v}_{1,2}=$ $(1 / \sqrt{2})\left[1 \pm e^{-j L a}\right]^{T}$. This allows (42) to be evaluated as

$$
\mathbf{R}_{T}^{\prime}=\eta\left[\begin{array}{ll}
c_{1} & c_{2} \\
c_{2}^{*} & c_{1}
\end{array}\right]
$$

with

$$
\begin{aligned}
& c_{1}=b_{1}^{2}+\left|b_{2}\right|^{2}+2 \operatorname{Re}\left\{\gamma b_{1} b_{2}^{*}\right\}, \\
& c_{2}=2 b_{1} b_{2}+\gamma b_{1}^{2}+\gamma^{*} b_{2}^{2}, \\
& b_{1}=\left[(1+|a|)^{-1 / 2}+(1-|a|)^{-1 / 2}\right] / 2, \\
& b_{2}=\left[(1+|a|)^{-1 / 2}-(1-|a|)^{-1 / 2}\right] \exp (j \angle a) / 2,
\end{aligned}
$$

where $\gamma$ is from (37). The eigenvalues of $\mathbf{R}_{T}^{\prime}$ are $\eta\left(c_{1} \pm\left|c_{2}\right|\right)$.

Because we have now constrained the radiated power to be constant and because our beamforming vector for $T=1$ is optimal, the effective gain becomes the ratio of the dominant eigenvalue of the transmit covariance matrix for two antennas

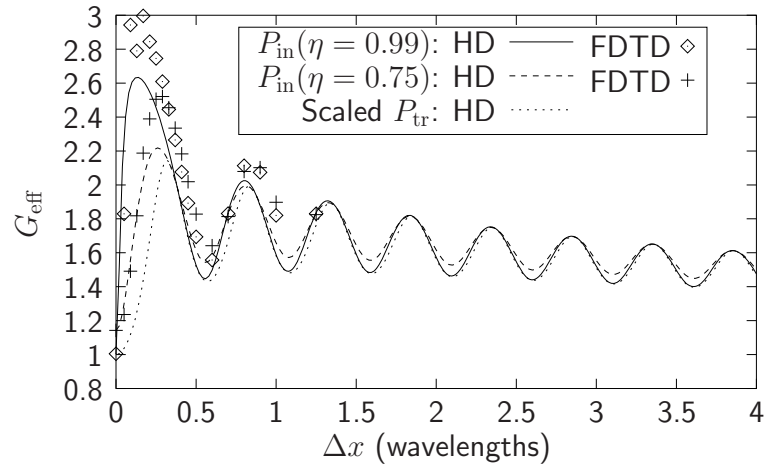

Fig. 9. Effective gain for the input-power constraint as a function of antenna spacing assuming a single departing cluster distributed according to the von Mises distribution for $\kappa=10$ and $\bar{\phi}=0$

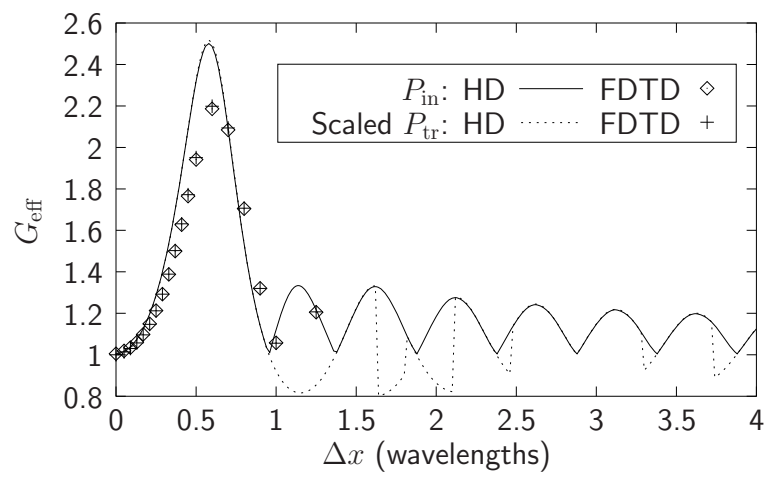

Fig. 10. Effective gain for the input-power constraint as a function of antenna spacing assuming a single departing cluster distributed according to the von Mises distribution for $\kappa=10$ and $\bar{\phi}=\pi / 2$

to that for a single antenna. Since for a single antenna this eigenvalue is equal to $\eta$, the effective gain of two antennas over the single element is $c_{1}+\left|c_{2}\right|$.

Figure 9 plots the effective gain versus antenna spacing for a single endfire von Mises cluster with $\kappa=10$ and different antenna efficiencies $(\eta)$. The effective gain for the traditional power constraint scaled according to radiated power is also plotted for comparison. The result shows that for lower antenna efficiencies the optimal and suboptimal solutions are almost equivalent. As the antenna efficiency increases, however, the optimal spacing approaches zero, indicating the existence of supergain solutions.

Figure 10 plots a similar result for a single broadside cluster with a fixed efficiency of $\eta=0.99$. As can be seen, the discontinuities in the effective gain have been removed by the input-power constraint. For narrow spacings, however, the two solutions are nearly identical.

\section{CONCLUSion}

This paper includes electromagnetic considerations in the analysis of correlated block-fading MIMO channels with covariance information. The formulation reveals that when antennas are closely spaced and exhibit mutual coupling, the traditional power constraint does not limit the radiated power, and therefore a new constraint is provided that explicitly limits the power radiated by the array. The analysis demonstrates that the addition of correlated transmit antennas can increase 
capacity only if the increase in system correlation is not offset by a corresponding increase in mutual coupling. For channels that result in this increased capacity, the capacity growth with increasing numbers of transmit elements can be enhanced or degraded by the coupling. Furthermore, for rapidly fading channels, the formulation indicates that antennas should not be placed as close together as possible as has been previously suggested. When the departing multipath has a strong directional bias relative to the transmit array orientation, spacings between 0.3 and 0.6 wavelengths may be optimal. When no such bias is present, the optimality of narrower spacing diminishes, and antennas may be placed arbitrarily far apart. In all cases, the new analysis shows that as the antenna spacing approaches zero, there is no increase in the system performance over that for a single antenna.

\section{REFERENCES}

[1] G. J. Foschini and M. J. Gans, "On limits of wireless communications in a fading environment when using multiple antennas," Wireless Personal Commun., vol. 6, pp. 311-335, Mar. 1998.

[2] G. G. Raleigh and J. M. Cioffi, "Spatio-temporal coding for wireless communication," IEEE Trans. Commun., vol. 46, pp. 357-366, Mar. 1998.

[3] I. E. Telatar, "Capacity of multi-antenna gaussian channels," AT\&T Bell Laboratories, Tech. Rep. Technical Report \#BL0112170-950615-07TM, 1995.

[4] V. Pohl, P. H. Nguyen, V. Jungnickel, and C. von Helmolt, "Continuous flat-fading MIMO channels: achievable rate and optimal length of the training and data phases," IEEE Trans. Wireless Commun., vol. 4, pp. 1889-1900, July 2005.

[5] A. Goldsmith, S. A. Jafar, N. Jindal, and S. Vishwanath, "Capacity limits of MIMO channels," IEEE Trans. Inf. Theory, vol. 21, pp. 684-702, June 2003.

[6] J. Wallace and M. Jensen, "Measurement and characterization of the time variation of indoor and outdoor MIMO channels at 2.4 and 5.2 GHz," in Proc. 2005 IEEE 62nd Veh. Technol. Conf., vol. 1, Sept. 25-28 2005

[7] T. L. Marzetta and B. M. Hochwald, "Capacity of a mobile multipleantenna communication link in Rayleigh flat fading," IEEE Trans. Inf. Theory, vol. 45, pp. 139-157, Jan. 1999.

[8] T. Fügen, J. Maurer, W. Sörgel, and W. Wiesbeck, "Characterization of multipath clusters with ray-tracing in urban MIMO propagation environments at $2 \mathrm{GHz}$," in Proc. 2005 IEEE 62nd Veh. Technol. Conf., vol. 1, Sept. 25-28 2005.

[9] E. A. Jorswieck and H. Boche, "Channel capacity and capacity-range of beamforming in MIMO wireless systems under correlated fading with covariance feedback," IEEE Trans. Wireless Commun., vol. 3, pp. 15431553, Sept. 2004.

[10] S. A. Jafar and A. Goldsmith, "Multiple-antenna capacity in correlated rayleigh fading with channel covariance information," IEEE Trans. Wireless Commun., vol. 4, pp. 990-997, May 2005.

[11] K. Yu, M. Bengtsson, B. Ottersten, D. McNamara, P. Karlsson, and M. Beach, "A wideband statistical model for NLOS indoor MIMO channels," in Proc. 2002 IEEE 55th Veh. Technol. Conf., vol. 1, May 6-9, 2002, pp. 370-374.

[12] IEEE Std 145-1993, IEEE Standard Definitions of Terms for Antennas, Antenna Standards Committee of the IEEE Antennas and Propagation Society, 1993.

[13] J. W. Wallace and M. A. Jensen, "Mutual coupling in MIMO wireless systems: A rigorous network theory analysis," IEEE Trans. Wireless Commun., vol. 3, pp. 1317-1325, July 2004.

[14] D. H. Staelin, A. W. Morgenthaler, and J. A. Kong, Electromagnetic Waves. Prentice-Hall, 1994.
[15] D. M. Pozar, Microwave Engineering. John Wiley \& Sons, 1998.

[16] A. Taflove and S. C. Hagness, Computational Electrodynamics: The Finite Difference Time Domain Method. Artech House, 2000.

[17] K. S. Yee, "Numerical solution of initial boundary value problems involving Maxwell's equations in isotropic media," IEEE Trans. Antennas Propag., vol. AP-14, pp. 302-307, 1966.

[18] M. Morris, M. Jensen, and J. Wallace, "Superdirectivity in MIMO systems," IEEE Trans. Antennas Propag., vol. 53, pp. 2850-2857, Sept. 2005.

[19] W. C. Jakes, Microwave Mobile Communications. IEEE Press, 1993.

[20] A. Abdi, J. Barger, and M. Kaveh, "A parametric model for the distribution of the angle of arrival and the associated correlation function and power spectrum at the mobile station," IEEE Trans. Veh. Technol., vol. 51, pp. 425-434, May 2002.

[21] V. Jungnickel, V. Pohl, and C. von Helmolt, "Capacity of MIMO systems with closely spaced antennas," IEEE Commun. Lett., vol. 7, pp. 361363, Aug. 2003.

[22] N. W. Bikhazi and M. A. Jensen, "The relationship between antenna loss and superdirectivity in MIMO systems," Brigham Young University, Tech. Rep. https://dspace.byu.edu/handle/1877/66, 2005.

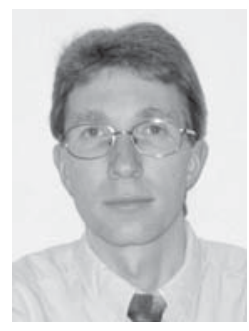

Jon W. Wallace (S'99, M'03) received the B.S (summa cum laude) and Ph.D. degrees in Electrical Engineering from Brigham Young University (BYU) in 1997 and 2002, respectively. From 1995 to 1997 he worked as an associate of Novell, Inc. in Provo, Utah. During 1997 he was a Member of Technical Staff for Lucent Technologies in Denver, Colorado. He received the National Science Foundation Graduate Fellowship in 1998 and worked as a graduate research assistant at BYU until 2002. From 2002 to 2003, he was with the Mobile Communications Group at the Vienna University of Technology, in Vienna, Austria, pursuing collaborative research in the area of wireless channel measurement and modeling. From 2003 to 2006 he was a research associate with the BYU Wireless Communications Laboratory, developing platforms for wideband MIMO channel sounding and real-time space-time coding. Dr. Wallace is currently an assistant professor of Electrical Engineering at Jacobs University Bremen, in Bremen, Germany. He is also serving as an associate editor of IEEE Transactions on Antennas and Propagation. His current research interests include wireless channel sounding and modeling, MIMO communications, cognitive radio, and UWB and frequency-agile systems.

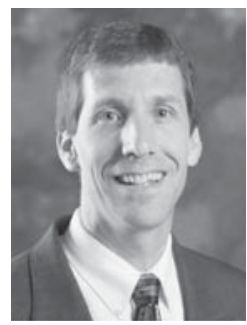

Michael A. Jensen (S'93, M'95, SM'01, F'08) received the B.S. (summa cum laude) and M.S degrees in Electrical Engineering from Brigham Young University (BYU) in 1990 and 1991, respectively, and the Ph.D. in Electrical Engineering at the University of California, Los Angeles (UCLA) in 1994. From 1989 to 1991 he was a graduate research assistant in the Lasers and Optics Laboratory at BYU. In 1990 he received a National Science Foundation Graduate Fellowship. From 1991 to 1994, he was a graduate student researcher in the Antenna Laboratory at UCLA. Since 1994, he has been at the Electrical and Computer Engineering Department at BYU where he is currently a Professor and Department Chair. His main research interests include antennas and propagation for personal communications, radar remote sensing, numerical electromagnetics, and optical fiber communications. He is a member of Eta Kappa $\mathrm{Nu}$ and Tau Beta Pi.

Dr. Jensen currently chairs the Joint Meetings Committee for the IEEE Antennas and Propagation Society. He has been an associate editor for the IEEE Transactions on Antennas and Propagation, a member of the society Administrative Committee, and Co-chair and Technical Program Chair for several symposia. He was awarded the H. A. Wheeler paper award in the IEEE Transactions on Antennas and Propagation in 2002, and the best student paper award at the 1994 IEEE International Symposium on Antennas and Propagation. 